\title{
In-situ ellipsometry measurements on the phase segregation of mixed halide lead perovskites
}

Annik Bernhardt, ${ }^{[a], ~[b], ~[c] ~ T h a r u s h i ~ D . ~ A m b a g a s p i t i y a, ~}{ }^{[a], ~[c] ~ M a r t i n ~ E . ~ K o r d e s c h, ~}{ }^{[c], ~[d]}$ Katherine Leslee A. Cimatu, [a], [c] Jixin Chen*, [a], [c]

[a] Department of Chemistry and Biochemistry, Ohio University, Athens, OH 45701, United States

[b] Department of Chemistry, University of Leipzig, 04103, Leipzig, Germany

[c] Nanoscale and Quantum Phenomena Institute, Ohio University, Athens, OH 45701, United States

[d] Department of Physics and Astronomy, Ohio University, Athens, OH 45701, United States

\begin{abstract}
Mixed halide lead perovskite such as methylammonium lead iodide bromides $\operatorname{MAPb}\left(\mathrm{Br}_{\mathrm{x}} \mathrm{I}_{1-\mathrm{x}}\right)_{3}$ have emerged as one of the most promising materials of future solar cells, offering high power conversion efficiencies and bandgap tunability. Among other factors, the reversible phase segregation under even low light intensities is still limiting their potential use. During this process, the material segregates locally into iodide-rich and bromide-rich phases, lowering the effective bandgap energy. While several studies have been done to illuminate the mechanism and suppression of phase segregation, fundamental aspects remain unclear. Phase compositions after segregation vary extensively between different studies and the exact amounts of phases often remain unmentioned. For iodide-rich phases, the end-point compositions at around $x=0.2$ are widely accepted but the proportion of the phase is difficult to measure. In this report, we observe the phase segregation using spectroscopic ellipsometry, a powerful, nondestructive technique that has been employed in the study of film degradation before. We obtained dynamic ellipsometric measurements from $x=0.5$ mixed halide lead perovskite thin films protected by a polystyrene layer under green laser light with a power density of $\sim 11 \mathrm{~W} / \mathrm{cm}^{2}$. Changes in the bandgap region can be correlated to the changes in composition caused by phase segregation, allowing for the kinetics to be observed. Time constants between $1.7( \pm 0.7) \times 10^{-3} \mathrm{~s}^{-1}$ for the segregation and $1.5( \pm 0.6) \times 10^{-4} \mathrm{~s}^{-1}$ for recovery were calculated. We expect ellipsometry to serve as a complementary technique to other spectroscopies in studying mixed-halide lead perovskites phase segregation in the future.
\end{abstract}

Keywords: Mixed-halide lead perovskite $\bullet$ phase separation $\bullet$ ellipsometry $\bullet$ kinetic measurement

With power conversion efficiencies reaching up to $25.5 \%$, perovskites have emerged as a promising material for the next generation of solar cells. ${ }^{[1]}$ Mixed halide perovskites offer, among others, the advantages of good bandgap tunability. ${ }^{[2]}$ Hoke et.al. observed reversible phase segregation in mixed 
bromide and iodide methylammonium lead perovskites $\mathrm{MAPb}\left(\mathrm{Br}_{x} \mathrm{I}_{1-x}\right)_{3}$ with a bromide content between $20 \%$ and $100 \%$ under illumination with light of various wavelengths and less than one sun intensity. They theorized the formation of small, iodide-rich domains. ${ }^{[2]}$ Since then there have also been research studies looking into the factors influencing phase segregation, its mechanism, and its influence on solar cell performance, which has been discussed elsewhere. ${ }^{[3,4]}$

There have been difficulties to quantify the phase segregation of mixed halide perovskites. From photoluminescence (PL) measurements which are most commonly employed, an estimation of the amounts of bromide-rich and iodide-rich phases are not easily available, since charge carriers rapidly transfer to the I-rich phase that dominates the PL spectrum. ${ }^{[5]}$ Hoke et al. estimated the amount of iodide-rich phase $(x=0.2)$ to be $1 \%$ from the photocurrent spectroscopy measurements of a mesoporous film in a solar cell device with 0.6 as the initial $x$. But x-ray diffraction (XRD) measurements of a thinfilm with the same composition yield 23\%. ${ }^{[2]}$ XRD measurements were also carried out by Duong et al. who observed segregation of $x=0.48$ into $x=1$ and $x=0.45$ with $90 \%$ of iodide-rich phase. ${ }^{[6]}$ Barker et al. ${ }^{[7]}$ observed the phase segregation with photothermal deflection spectroscopy and arrived at values of $5 \%$ for the iodide-rich phase for both $x=0.4$ and $x=0.6$ thin-film samples. Yoon et al. ${ }^{[5]}$ observed the segregation of $x=0.43$ by absorption measurements with $18 \%$ of segregation into $x=0.32$ and $x=1$ estimated from a decrease in the absorption of the parent perovskite. A much lower amount of $2 \%$ of segregation estimated from an increase in absorption for the I-rich phase was explained by distorting effects of tail absorption. Several studies have concluded that besides the I-rich and Br-rich regions, a large part of the sample remains unchanged at the original composition. ${ }^{[8,9]}$

Typically, the phase segregation results in an exponential growth of the photoluminescence intensity for the I-rich phase and an exponential decay of the absorption of the original phase respectively, that saturates at a certain point. The rate constants of segregation range from $0.1-0.01 \mathrm{~s}^{-1}$ for photoluminescence measurements and one magnitude smaller for absorption measurements. The rate constants of the recovery range from $10^{-3}$ to $10^{-5} \mathrm{~s}^{-1}$. These constants are also dependent on the excitation wavelength and intensity, as well as film thickness and quality. ${ }^{[3]}$

Ellipsometry is an optical technique that measures the dielectric properties of thin films. It has previously been employed in the study of lead halide perovskites reviewed recently by Li et.al. ${ }^{[10]}$ The method offers several advantages including fast, non-destructive measurements that are sensitive to optical constants and the thickness of layered samples. Because it measures the reflective differences among differently polarized light at various wavelengths and angles, it is insensitive to environmental disturbances and is accurate, robust, reproducible, and sensitive to changes in phase, bandgap, and thickness. For this reason phase segregation of perovskite samples protected by a polymer film can be 
observed under atmospheric conditions. Phenomena like the change in optical constants with halide composition $^{[11]}$, the effect of voids and roughness ${ }^{[12]}$, and degradation by atmospheric conditions including light ${ }^{[13]}$, hydration ${ }^{[14]}$, and temperature ${ }^{[15]}$ of halide lead perovskites have been studied previously. To the best of our knowledge, no study on the phase segregation of mixed halide perovskites using ellipsometric measurements has been done so far.

In the following communication, we present the observation of phase segregation with dynamic ellipsometric measurements. This method enables continuous measurement of the change in the composition during the segregation and recovery process.

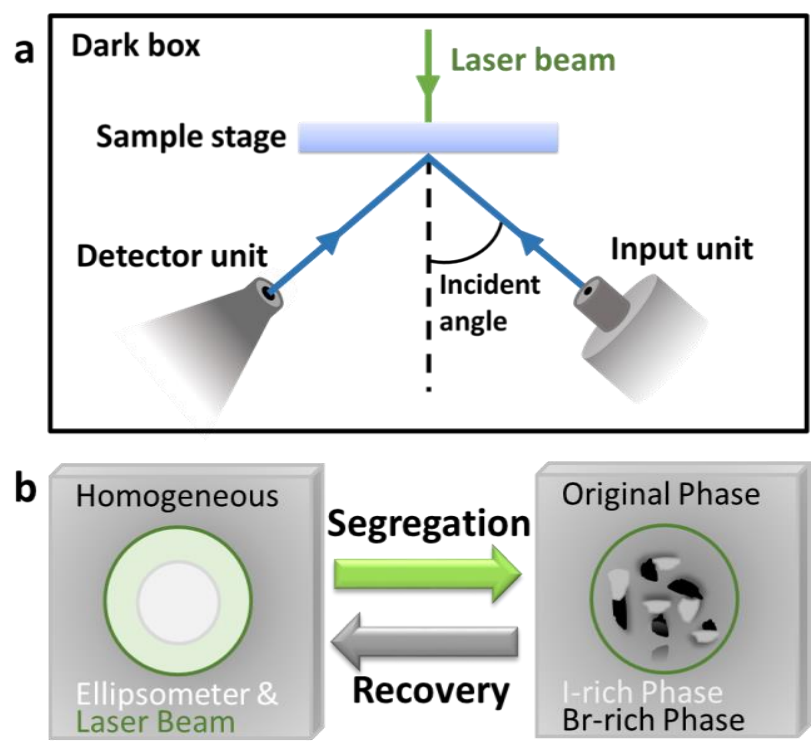

Figure 1. (a) Scheme of the ellipsometric measuring setup with safety box and (b) scheme of the phase segregation. The white spot represents the ellipsometry illumination light and the green spot represents the laser illumination light with diameter $\sim 2.5 \mathrm{~mm}$.

Perovskite thin films were synthesized via solvent-washing, spin-coating, and encapsulating with polystyrene with its protocol described elsewhere. ${ }^{[16]}$ The as-synthesized mixed halide perovskites with $x=0.1, x=0.5$, and $x=1$ were characterized by both photoluminescence and UV-Vis spectroscopy (Supporting Information Figure S2). The bandgap of samples with $x=0.5$ is ca. $665 \mathrm{~nm}(1.86 \mathrm{eV})$, the emission peak is at $656 \pm 5 \mathrm{~nm}$ and starts splitting into two peaks within 3 seconds of illumination. After 30 min of illumination, the photoluminescence peak of $x=0.5$ has shifted to $740 \pm 2 \mathrm{~nm}$, a value close to samples with a nominal composition $x=0.1$ with a peak at $744 \pm 3 \mathrm{~nm}$.

Samples with $x=0.5$ were further characterized. Scanning electron microscopy measurements (Figure S3) show uniform samples with some pinholes mainly below $10 \mathrm{~nm}$ but up to $80 \mathrm{~nm}$ in size. The grain size was estimated to be $56 \pm 13 \mathrm{~nm}$ (Figure S3). An XRD spectrum in Figure $\mathbf{S 4}$ shows 
expected 110 and 200 plane peaks. Ellipsometric measurements of the samples as exemplified in Figure S5 has revealed thicknesses of $195 \pm 4 \mathrm{~nm}$ for the perovskite layer and $1042 \pm 14 \mathrm{~nm}$ for the polystyrene layer. Average values of all fitting parameters for the three samples are given in the Table S2. A control sample on Si substrate had a thickness of $869 \mathrm{~nm}$ and $193 \mathrm{~nm}$ for polystyrene and perovskite layers respectively, with a thickness non-uniformity of $7 \%$ as determined by the ellipsometric model fitting. Atomic force microscopy measurements (Figure S6) of the same sample showed consistent corresponding thicknesses of $825 \pm 48 \mathrm{~nm}$ and $199 \pm 19 \mathrm{~nm}$ respectively. Thickness non-uniformity estimations ranged from $2 \%-9 \%$ for the polystyrene layer and $5 \%-10 \%$ for the perovskite layer.

Laser light shining through the backside of $x=0.5$ samples spun-coat on glass coverslips initiated phase segregation. The power density of the laser illumination is estimated to be $11 \mathrm{~W} / \mathrm{cm}^{2}$. The setup and separation scheme are provided in Figure 1. The laser beam was aimed such that it is focused on the same spot as the ellipsometer beam. The change in composition leads to the peak in the amplitude signal of ellipsometry $(\Psi)$ within the bandgap region to decrease and shift (Figure 2a and $2 \mathbf{b}$ ) most prominently at $50^{\circ}$ and between 600 and $650 \mathrm{~nm}$ depending on sample thickness (Figure S7). Under the laser light, ellipsometric measurements of samples with composition $x=1$ and $x=0.1$ both had negligible changes (Figure S9). Thus, combining with the results from earlier studies of these samples ${ }^{[2,5-7,9,16,17]}$, we conclude that the change in the ellipsometric measurements is due to the phase change instead of other effects such as changes in temperature, light scattering, or polymer films.

The resulting mixture of phases during segregation and recovery of $x=0.5$ samples was modeled by a Bruggemann effective medium approximation of $x=0.1, x=0.5$, and $x=1$. The percentage amount of each phase $p_{x}$ was calculated assuming the decreased $x=0.5$ portion is transferred to $x=1$ and $x=0.1$ with equations (1) and (2) based on the stoichiometry of the phase segregation:

$$
\begin{aligned}
& p_{1}=\frac{(0.5-0.1) \cdot\left(100 \%-p_{0.5}\right)}{1-0.1} \\
& p_{0.1}=100 \%-p_{1}-p_{0.5}
\end{aligned}
$$

The predicted value of $\Psi$ at the observed wavelength in the model was fit to $p_{0.5}$ between $100 \%$ and $50 \%$ with a quadratic function (Figure 2c). This calculation is carried out for each measurement using the initial spectrum time $\mathrm{t}=0$ as the calibration point This also serves to remove any possible effect of roughness and interface variations among different samples with the same or different $\mathrm{Br}$ contents, i.e. the kinetics observed will be only from the change of the phases of the same sample rather than from other effects. From this data treatment, a decrease of $p_{0.5}$ under laser light from $100 \%$ to $77 \% \pm 4 \%$ during measurement times ranging from 100-200 minutes was observed. This corresponds to $10 \%$ of bromide-rich phase and $13 \%$ of iodide-rich phase. Nine recovery measurements between 1000 and 1500 
min in the dark revealed an increase of $p_{0.5}$ to $99 \% \pm 2 \%$. The existence of endpoints above $100 \%$ is from the error of the measurements.

The segregation and recovery processes were fit by exponential decay and growth functions respectively. The time constants were found to be $1.7( \pm 0.7) \times 10^{-3} \mathrm{~s}^{-1}$ for the segregation process and $1.5( \pm 0.6) \times 10^{-4} \mathrm{~s}^{-1}$ for the recovery. One exemplary measurement with fitting is shown in Figure 2d. For the values of all measurements see Tables S3, S4, and S5 in the Supporting Information. The recovery kinetics is poorly fitted by the single exponential decay function. A double-exponential curve fits all but one of the curves better indicating a more complicated recovery mechanism with an amplitude-averaged rate constant calculated as $2.01( \pm 1.26) \times 10^{-3} \mathrm{~s}^{-1}$. The parameters of these fits are listed in Table S6. The phase segregation rates are slower than those measured with photoluminescence and the recovery rates are consistent with the literature values.
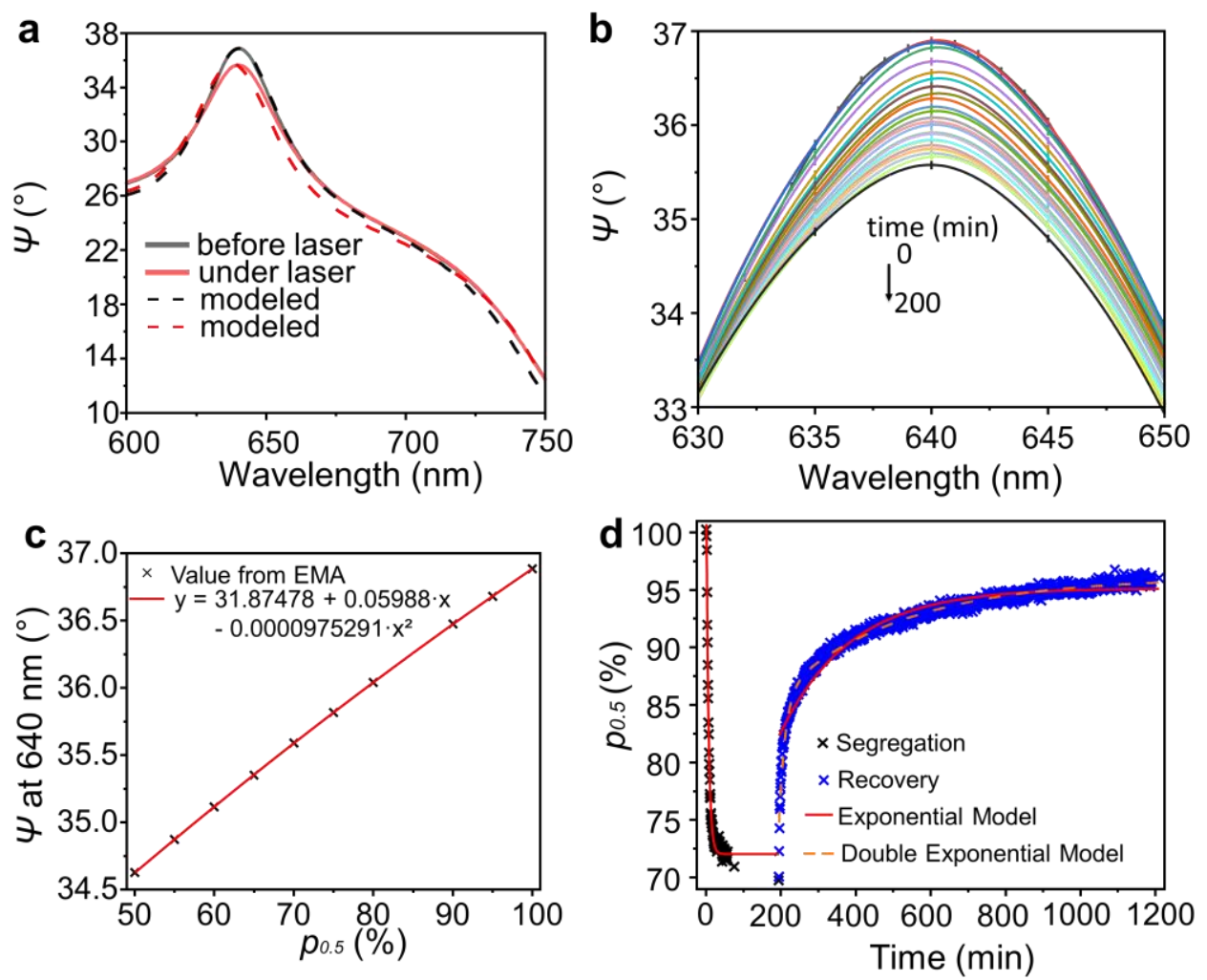

Figure 2. Ellipsometric amplitude parameter $\Psi$ of $x=0.5$ at $50^{\circ}$ (a) before laser exposure and under laser light including modeling by fitting algorithm, (b) relevant peak at selected times under laser exposure (peaks are interpolated between measurement points by Akima Splines, time labeled in Figure S7), (c) The dependence of the calculated value of $\Psi$ on the content of $p_{0.5}$ in a mixture of perovskite phases are fitted with a quadratic function, (d) segregation and recovery illustrated by the fraction of $x=0.5$ phase $p_{0.5}$ over time and fitted by exponential functions. 
In summary, ellipsometry offers a simple and non-destructive method to quantify the kinetics of phase segregation and the amount of the segregated phases without problems regarding quantum efficiency in PL measurements or shape and amorphous effects in XRD measurement. Thus, we expect it to serve as a complementary technique to other spectroscopies in studying mixed-halide lead perovskites phase segregation. It cannot be completely excluded that the ellipsometry measurements are affected by changes to the morphology of the sample e.g. the formation of voids or changes to thickness non-uniformity. Nevertheless, the relatively low noise and complete recovery (within $2 \%$ error) of original measurements demonstrate its stability in quantifying phase changes.

\section{Acknowledgements}

The authors thank NQPI and Prof. Eric Stinaff at Ohio University for sharing instruments.

\section{References}

[1] "Best Research-Cell Efficiency Chart", can be found under https://www.nrel.gov/pv/cellefficiency.html, 2021.

[2] E. T. Hoke, D. J. Slotcavage, E. R. Dohner, A. R. Bowring, H. I. Karunadasa, M. D. McGehee, Chemical science 2015, 6, 613.

[3] M. C. Brennan, A. Ruth, P. V. Kamat, M. Kuno, Trends in Chemistry 2020, 2, 282.

[4] a) R. A. Kerner, Z. Xu, B. W. Larson, B. P. Rand, Joule 2021, 5, 2273; b) Y. Guo, X. Yin, W. Que, W. Liu, H. Liu, J. Mater. Chem. C 2020, 8, 14626; c) A. J. Knight, L. M. Herz, Energy Environ. Sci. 2020, 13, 2024; d) Y. Wang, X. Quintana, J. Kim, X. Guan, L. Hu, C.-H. Lin, B. T. Jones, W. Chen, X. Wen, H. Gao et al., Photon. Res. 2020, 8, A56; e) M. C. Brennan, S. Draguta, P. V. Kamat, M. Kuno, ACS Energy Lett. 2018, 3, 204; f) C. G. Bischak, A. B. Wong, E. Lin, D. T. Limmer, P. Yang, N. S. Ginsberg, The journal of physical chemistry letters 2018, 9, 3998; g) M. Lai, A. Obliger, D. Lu, C. S. Kley, C. G. Bischak, Q. Kong, T. Lei, L. Dou, N. S. Ginsberg, D. T. Limmer et al., Proceedings of the National Academy of Sciences of the United States of America 2018, 115, 11929.

[5] S. J. Yoon, S. Draguta, J. S. Manser, O. Sharia, W. F. Schneider, M. Kuno, P. V. Kamat, ACS Energy Lett. 2016, 1, 290.

[6] T. Duong, H. K. Mulmudi, Y. Wu, X. Fu, H. Shen, J. Peng, N. Wu, H. T. Nguyen, D. Macdonald, M. Lockrey et al., ACS applied materials \& interfaces 2017, 9, 26859. 
[7] A. J. Barker, A. Sadhanala, F. Deschler, M. Gandini, S. P. Senanayak, P. M. Pearce, E. Mosconi, A. J. Pearson, Y. Wu, A. R. Srimath Kandada et al., ACS Energy Lett. 2017, 2, 1416.

[8] A. J. Knight, J. Borchert, R. D. J. Oliver, J. B. Patel, P. G. Radaelli, H. J. Snaith, M. B. Johnston, L. M. Herz, ACS Energy Lett. 2021, 6, 799.

[9] X. Tang, M. van den Berg, E. Gu, A. Horneber, G. J. Matt, A. Osvet, A. J. Meixner, D. Zhang, C. J. Brabec, Nano letters 2018, 18, 2172.

[10]H. Li, C. Cui, X. Xu, S. Bian, C. Ngaojampa, P. Ruankham, A. P. Jaroenjittchai, Solar Energy 2020, 212,48 .

[11] a) S. Fujimoto, T. Fujiseki, M. Tamakoshi, A. Nakane, T. Miyadera, T. Sugita, T. N. Murakami, M. Chikamatsu, H. Fujiwara in Springer Series in Optical Sciences (Eds.: H. Fujiwara, R. W. Collins), Springer International Publishing, Cham, 2018, pp. 471-493; b) P. F. Ndione, Z. Li, K. Zhu, J. Mater. Chem. C 2016, 4, 7775.

[12] a) M. S. Alias, I. Dursun, M. I. Saidaminov, E. M. Diallo, P. Mishra, T. K. Ng, O. M. Bakr, B. S. Ooi, Optics express 2016, 24, 16586; b) X. Ziang, L. Shifeng, Q. Laixiang, P. Shuping, W. Wei, Y. Yu, Y. Li, C. Zhijian, W. Shufeng, Du Honglin et al., Opt. Mater. Express 2015, 5, 29; c) K. Ghimire, A. Cimaroli, F. Hong, T. Shi, N. Podraza, Y. Yan in 2015 IEEE 42nd Photovoltaic Specialist Conference (PVSC), IEEE, 62015, pp. 1-5; d) H. Fujiwara, S. Fujimoto, M. Tamakoshi, M. Kato, H. Kadowaki, T. Miyadera, H. Tampo, M. Chikamatsu, H. Shibata, Applied Surface Science 2017, 421, 276.

[13]A. Marronnier, H. Lee, H. Lee, M. Kim, C. Eypert, J.-P. Gaston, G. Roma, D. Tondelier, B. Geffroy, Y. Bonnassieux, Solar Energy Materials and Solar Cells 2018, 178, 179.

[14]A. M. A. Leguy, Y. Hu, M. Campoy-Quiles, M. I. Alonso, O. J. Weber, P. Azarhoosh, M. van Schilfgaarde, M. T. Weller, T. Bein, J. Nelson et al., Chem. Mater. 2015, 27, 3397.

[15] Y. Jiang, A. M. Soufiani, A. Gentle, F. Huang, A. Ho-Baillie, M. A. Green, Appl. Phys. Lett. 2016, 108,61905 .

[16] J. R. Vicente, M. E. Kordesch, J. Chen, Journal of Energy Chemistry 2021, 63, 8.

[17] J. R. Vicente, J. Chen, The journal of physical chemistry letters 2020, 11, 1802. 


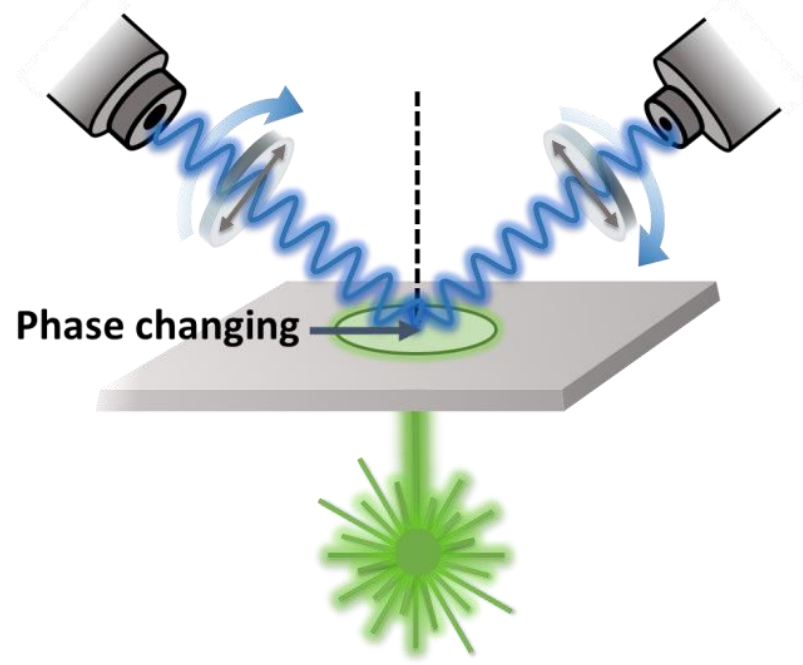

